\title{
Strain effects on the valence band structure, optical transitions, and light gain spectra in zinc-blende GaN quantum wells
}

\author{
L.O. Lokot \\ V. Lashkaryov Institute for Semiconductor Physics, Department of Theoretical Physics, \\ 41, prospect Nauky, 03028 Kyiv, Ukraine, e-mail: lyuba.lokot@gmail.com
}

\begin{abstract}
A study for the effects of size quantization and strain effects on the valence band spectra, the interband matrix elements, and the light gain spectrum in zinc-blende GaN quantum wells is presented. In the framework of the effective mass theory, the Schrödinger equation is solved for the valence bands with a $3 \times 3$ block Hamiltonian. The results are illustrated for the $\mathrm{GaN} / \mathrm{Al}_{0.19} \mathrm{Ga}_{0.81} \mathrm{~N}$ quantum well. It is shown, that the biaxial strain causes quite significant changes to the gain spectra in spatially confined structures. It is shown, that laser effect is suppressed with arising of the circular loop of valence band maxima in the heterostructure under the tensile strain, while under the compressive strain, the stimulated emission is pronounced. Our results show the internal strain effects are important in optical properties of $\mathrm{GaN}$ and associated quantum well structures.
\end{abstract}

Keywords: strain effect, valence band structure, optical transition, light gain spectra, zinc-blende $\mathrm{GaN}$, quantum well.

Manuscript received 04.08.08; accepted for publication 20.10.08; published online 11.11.08.

Direct wide band gap group III-nitride semiconductors based on GaN and its alloys have received great attention due to prospective applications in optoelectronic devices such as light-emitting diodes and lasers at green-blue and near-ultraviolet wavelengths, solar-blind photodetectors [1, 2]. A number of ultraviolet light-emitting diodes [3-8], and laser diodes [9-15] already have been demonstrated. However, nitride structures and devices are still in the developmental stage.

Internal strain effects in thin films become increasingly important in modern solid state technology. An important problem in growing GaN on crystalline substrates $\mathrm{Si}, \mathrm{SiC}, \mathrm{GaAs}, \mathrm{ZnO}$ and sapphire is an internal strain. Internal strains are related to the large lattice mismatch and the difference in the thermal-expansion coefficients of the epitaxial layer and a substrate. They can cause large biaxial strains in the epitaxial layers. Biaxial strains can be compressive or tensile depending on the crystalline substrate material [16-18]. The internal strain effects are studied in this paper. We present studies of the influence of biaxial strain on the valence band spectra of zinc-blende GaN/AlGaN quantum well of width $w$ perpendicular to the growth direction (001) and located at $-w / 2<z<w / 2$. Under biaxial strain, the transverse components of strain are proportional to the difference in lattice constants $a_{0}$, also depend on $\mathrm{Al}$ content $x$ :

$\varepsilon_{x x}=\varepsilon_{y y}=x\left(a_{0}^{\mathrm{AlN}}-a_{0}^{\mathrm{GaN}}\right) / a_{0}^{\mathrm{GaN}}=-0.029 x<0$,

when as $\varepsilon_{x y}=\varepsilon_{y z}=\varepsilon_{x z}=0$ [19]. The longitudinal component of strain can be expressed as $\varepsilon_{z Z}=-2\left[C_{12} / C_{11}\right] \varepsilon_{x x}$, where $C_{12}$ and $C_{11}$ are the elastic constants. Since $\varepsilon_{x x}<0$ then the lattice mismatch causes a compressive strain of the quantum well. Situations under which, the crystalline substrate causes a tensile strain of the quantum well are studied. To compare the role of the compressive and the tensile biaxial strain effects, we consider unstrained thin film GaN.

In this paper, we present a quantitative analysis of strain and confinement effects on holes in zinc-blendes.

We consider an electron that being initially in the conduction band emits a photon and ends in the valence band top. To describe the emission or absorption processes, the energies as well as wave functions of the lowest conduction subband and the valence subbands are calculated. The strain dependence of both matrix elements for dipole optical interband transitions and the light gain spectra in zinc-blende $\mathrm{GaN}$ quantum wells have been derived. 
The point group of the zinc-blende structure is identical to the elements of the point group of a tetrahedron which is denoted by $T_{d}$. The space group of the zinc-blende structure is symmorphic and is denoted by $T_{d}^{2}$ [20]. The bonding between nearest neighbors in the würtzite crystal is also tetrahedral. The configuration of nearest neighbors of the first coordination sphere in the würtzite lattice coincides with that in zinc-blende structures under a relatively small deformation in the (111) direction [21]. For this reason, the physical background for the cubic approximation is based on similarity between the (0001) axis in the würtzite structure and (111) direction in the cubic crystal [21].

The space group of the würtzite structure is $C_{6 v}^{4}$. The cohesive energy of the würtzite structure is very close to that of the zinc-blende structure [20]. For that reason, GaN can crystallize in both zinc-blende and würtzite polytypes.

It is known [20-23], that the valence-band spectrum at the $\Gamma$ points originates from sixfold degenerate $\Gamma_{15}$ state. In zinc-blende structures, the $\Gamma_{15}$ level splits by the spin-orbit interaction, forming the fourfold degenerate $\Gamma_{8}$ heavy $(\mathrm{HH})$ and light $(\mathrm{LH})$ holes levels and double degenerate $\Gamma_{7}$ spin split-off (SH) holes level. Under the action of the hexagonal crystal field and the spin-orbit interaction in würtzite crystals, $\Gamma_{15}$ splits lead to the formation of three spin degenerate levels: $\Gamma_{9}$, upper $\Gamma_{7}$, and lower $\Gamma_{7}$ levels which are denoted by heavy holes, light holes and split-off holes, respectively. Conduction-band states in the vicinity of Brillouin zone center are spin degenerate and characterized by a single effective mass for cubic symmetry and two effective mass parameters for hexagonal symmetry.

The Hamiltonian for the würtzite valence band which accounts for the interaction of $\Gamma_{7}, \Gamma_{7}$, and $\Gamma_{7}$ levels has been derived within the $k p$ method [22]. Later, a derivation of the würtzite Hamiltonian based on the method of invariants with including the effects of strain on the hole spectra has been proposed [21, 24]. A transformation of the Hamiltonian written in the basis $|1, m\rangle|1 / 2, s\rangle$ to the basis of angular momenta $3 / 2$ and $1 / 2$ with the spin-orbit split-off band included has been performed in papers [23, 25]. The basis of angular momenta $3 / 2$ and $1 / 2$ is frequently used for the $6 \times 6$ Luttinger-Kohn Hamiltonian for zinc-blende structures. Using a unitary transformation, a more convenient block-diagonal form with two $3 \times 3$ blocks in the framework of the envelope function formalism has been proposed [23, 25, 26]. Latter approachs have been used in this work. Here we use the effective-mass parameters, spin-orbit splitting energy, and deformation potential parameters as in the papers [23, 27, 28].

We consider the pseudomorphically strained zincblende $\mathrm{GaN} / \mathrm{Al}_{0.19} \mathrm{Ga}_{0.81} \mathrm{~N}$ quantum well of width $5.2 \mathrm{~nm}$.
We assume a rectangular form of potential for the quantum well.

The results of numerical calculations of the valence band spectra and the $k$-dependence of the matrix elements as a function of the wave vector $k_{t}=\left\{k_{x}, k_{y}\right\}$, which lies in the plain of the quantum well, are presented in Figs 1 to 3 . Here for all the structures, the two highest hole bands are the heavy hole band and light hole one.

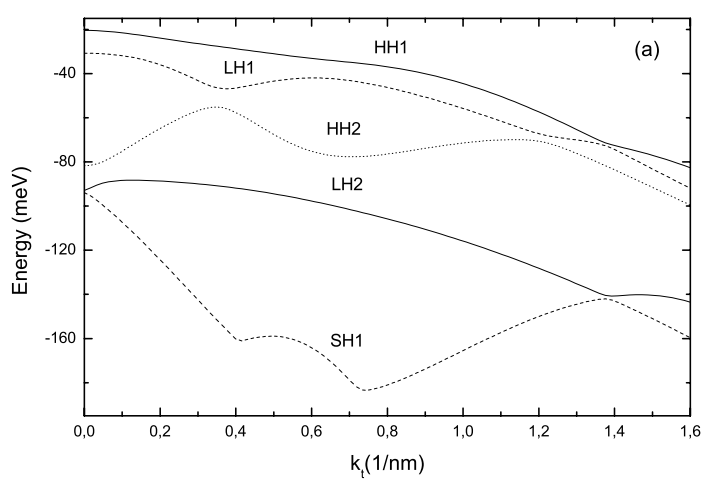

1a

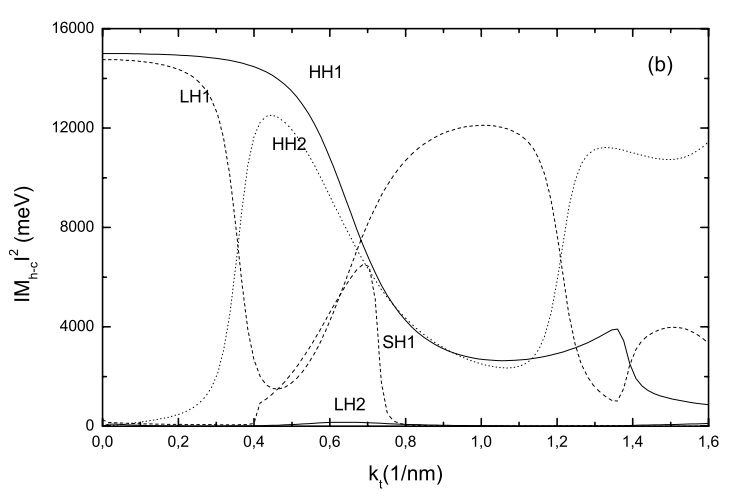

1b

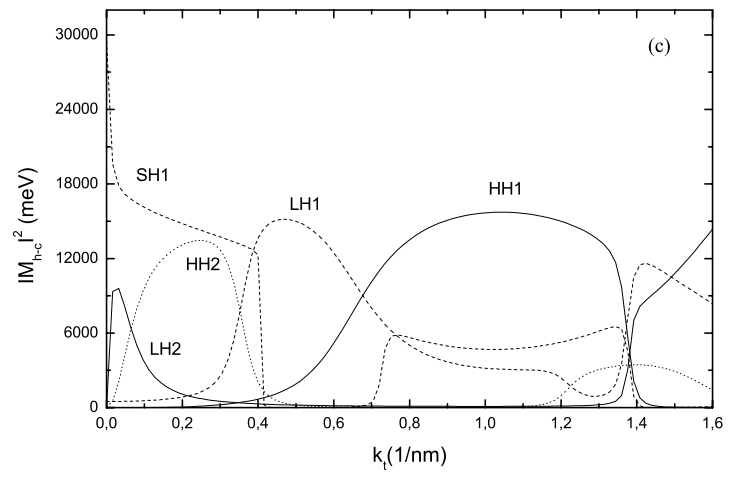

1c

Fig. 1. Unstrained thin film GaN: (a) the valence subband structure; the momentum matrix elements for (b) $x$ (or $y$ ) polarization and (c) z polarization. 
To clarify the role of compressive and tensile biaxial strain effects, we consider the unstrained thin film GaN. In Fig. 1, we show the valence-band structure and the $k$-dependence of the matrix elements. Fig. 1a shows that each band consists of a mixture of heavy hole, light hole and spin-orbit split hole states.

It is known that the $p$-like sixfold degenerated valence band at the $\Gamma$ point is separated into a fourfold degenerated $\Gamma_{8}$ state and a twofold degenerated $\Gamma_{7}$ state by the spin-orbit coupling. The value of this separation is the spin-orbit splitting energy, which is defined from the matrix element of the spin-orbit interaction Hamiltonian between the atomic orbitals and is usually referred to as the spin-orbit splitting width. The degeneracy is governed by the cubic symmetry. In the case when the quantum well is grown along the (001) direction, the crystal symmetry is changed to the tetragonal [29]. This implies removing the degeneracy of $\Gamma_{8}$ state. It is shown in Fig. 1a.

In Fig. 2, we show the valence-band structure and the $k$-dependence of matrix elements of the quantum well under the compressive biaxial strain. Components of strain are equal: $\varepsilon_{x x}=-0.0054, \varepsilon_{z z}=0.0063$. This strain is consistent with the aluminum content $19 \%$. In Fig. 2a, one can see that the compressive strain causes a downward shift of the valence bands. Such behavior agrees with the calculations of strain effects on the valence band structure in würtzite $\mathrm{GaN}$ quantum wells for nitride-based devices, which are fabricated on (0001) sapphire substrates [30].

We consider optical transitions between the initial and final states such as: with angular momentum $J=3 / 2$ and the magnetic quantum numbers $m_{j}= \pm 3 / 2, \quad m_{j}= \pm 1 / 2$ as well as with $J=1 / 2$, $m_{j}= \pm 1 / 2$ of the valence band and with $J=1 / 2$, $m_{j}= \pm 1 / 2$ of the conduction band. Transitions from the valence band states $m_{j}= \pm 1 / 2$ obey selection rules such as $\Delta m=0$ and $\Delta m= \pm 1$, therefore they have both the $x$ (or $y$ ) and $z$ light polarizations. Transitions from the valence band states $m_{j}= \pm 3 / 2$ obey selection rules $\Delta m= \pm 1$, therefore they have only the $x$ (or $y$ ) polarization [31].

Under the compressive strain, an increase in the aluminum content is accompanied with both an increase of the splitting width between the heavy hole and light hole bands and a decrease of valence-band mixing effects. Consider transitions from the heavy hole band. Figs 1 and 2 clearly indicate that the matrix elements have the stricter contribution of $x$ (or $y$ ) light polarization as goes from the unstrained thin film to the strained heterostructure. Thus, in the case of the compressively strained quantum well, the matrix elements have the strict $x$ (or $y$ ) light polarization. Such behavior agrees with the calculations [21, 32,33] of the momentum matrix elements in crystals of würtzite symmetry and associated quantum well structures, in which the considered transitions are allowed for the $x$ (or $y$ ) light polarization, while for the $z$ light polarization are forbidden.

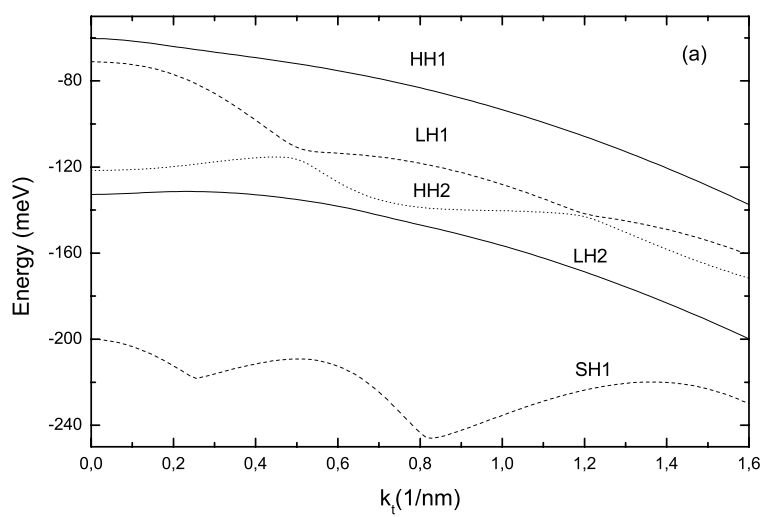

2a

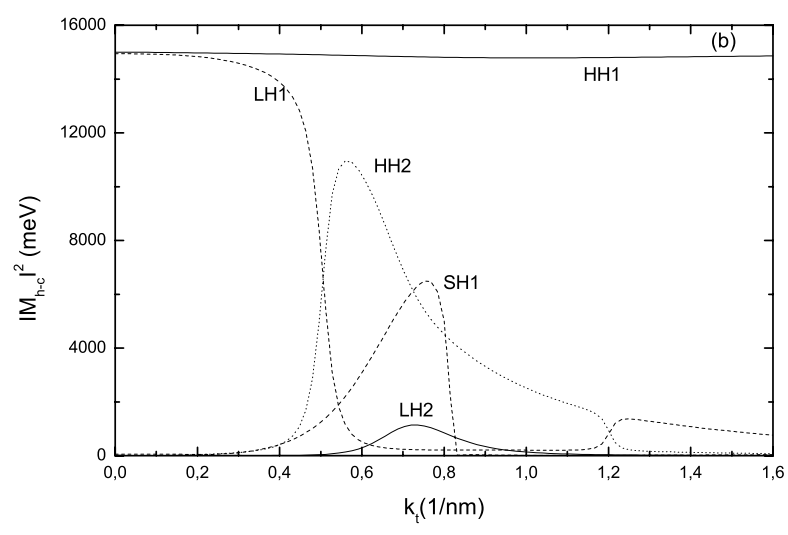

2b

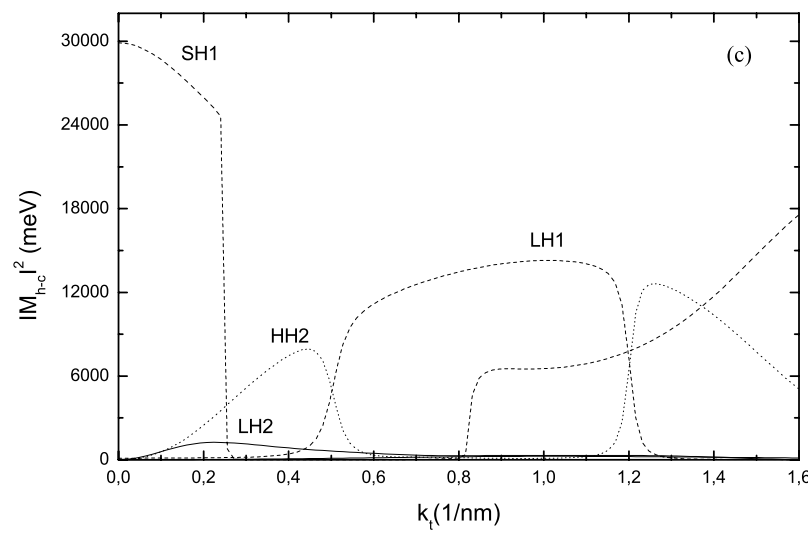

2c

Fig. 2. GaN/AlGaN quantum well with the compressive biaxial strain $\varepsilon_{x x}=-0.54 \%$ : (a) the valence subband structure; the momentum matrix elements for (b) $x$ (or $y$ ) polarization and (c) z polarization. 
In Fig. 3, we show the valence band structure and $k$-dependence of the matrix elements for the quantum well under the tensile biaxial strain. The strain components are equal: $\varepsilon_{x x}=0.0054, \varepsilon_{z z}=-0.0063$. In Fig. 3a, one can see that the tensile strain causes both strong transformation of hole effective mass and a decrease of the splitting width due to an upward shift of the light hole valence band to the top of the heavy hole one. The former leads to arising of the large negative mass at the Brillouin zone center, while the latter results in arising of the casual twofold degeneration of double spin degenerated heavy hole and light hole states at the Brillouin zone center. We show that a fourfold degeneration of the valence band states at the Brillouin zone center may be found in zinc-blende (001) GaN quantum well with the former deriving double spin degeneration of heavy hole and light hole states and the latter under the action of the tensile strain in addition to the casual twofold degeneration. In general, arising of the degeneracy causes an increase of the density of states. The light hole band can be shifted upward above of the heavy hole band with the tensile strain increase. This implies removing the fourfold degeneration of valence band states. Thus, at $\varepsilon_{x x}>0.0054$ the highest quantized hole subband is the light band. It is expected that the density of states is changed by the action of strain.

Such behavior qualitatively agrees with the calculations of internal strain effects on the valence band structure of $\mathrm{Si}_{1-\mathrm{x}} \mathrm{Ge}_{\mathrm{x}}$ [34].

In zinc-blende $\mathrm{GaN}$, the states of the light hole band are composed of the $|X \pm i Y\rangle$ characters as well as the $|Z\rangle$ character. Comparison of Figs 1 and 3 shows an increase of presence of the $|Z\rangle$ state in the light hole band as one moves from the unstrained thin film to the strained heterostructure, in which $\varepsilon_{x x}=0.0054$. There is more the $|Z\rangle$ state in the light hole band in the strained heterostructure, than in the thin film. Therefore, in the strained quantum well with $\varepsilon_{x x}>0.0054$, the states of the highest-lying light hole band are almost composed of the $|Z\rangle$ character, which yields the strong matrix element for the $\mathrm{z}$ light polarization.

Although both the compressive and the tensile biaxial strains were studied, only zinc-blende GaN quantum well under the tensile strain exhibits a region of the spectrum with negative effective mass and the strong modification of the matrix elements for the $z$ light polarization. The density of states, carrier population inversion, matrix elements, the light gain spectrum vary notably with arising of the spectral region with negative effective mass at the Brillouin zone center.

Understanding the influence of internal strain effects under the lattice mismatch on laser gain properties should help towards improving the laser performance and optimal device configurations.

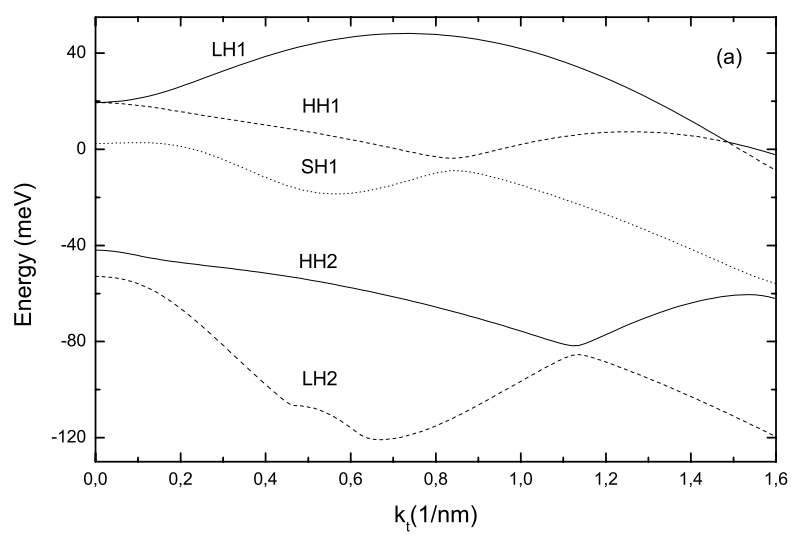

$3 a$

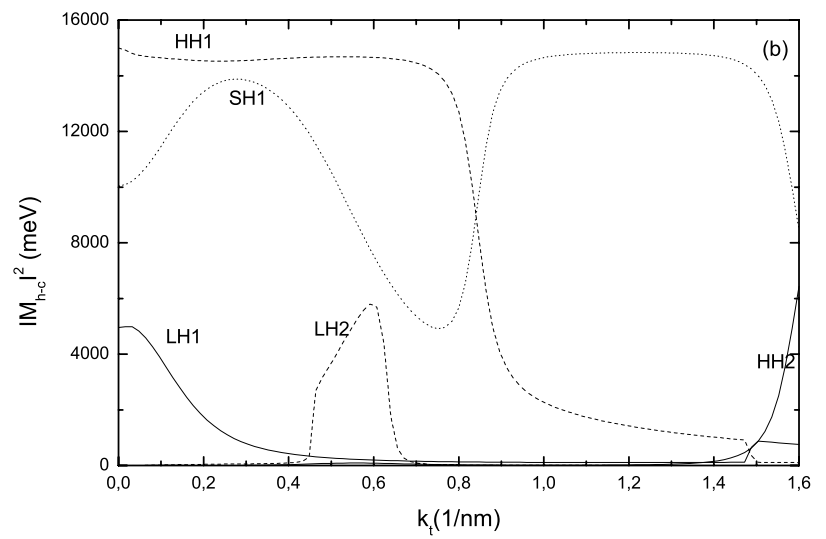

$3 \mathbf{b}$

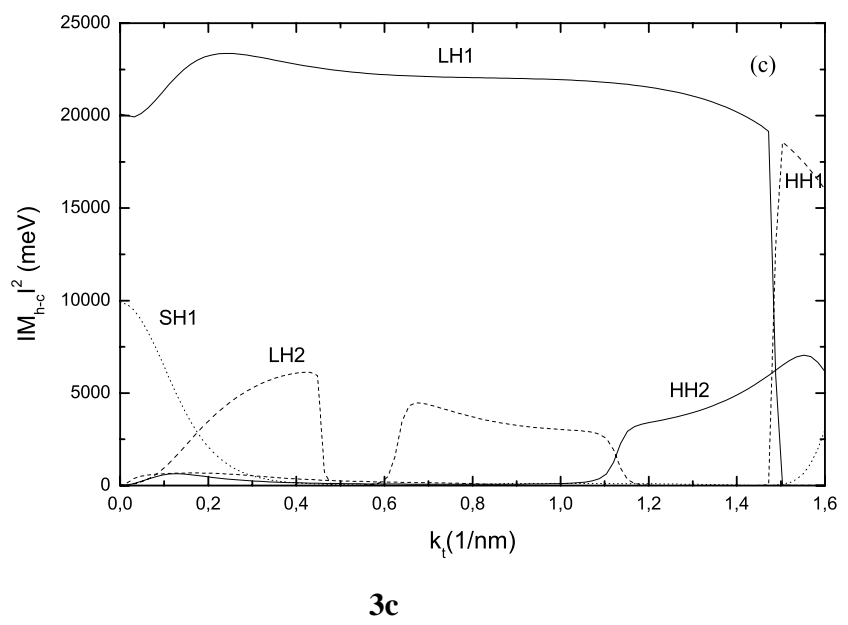

Fig. 3. GaN/AlGaN quantum well with tensile biaxial strain $\varepsilon_{x x}=0.54 \%$ : (a) the valence subband structure; the momentum matrix elements for (b) $x$ (or $y$ ) polarization and (c) $z$ polarization. 
In Fig. 4, we show the energy-dependence of the light gain coefficient of quantum well with the carrier concentration $5 \times 10^{12} \mathrm{~cm}^{-2}$ at the temperature $4.4 \mathrm{~K}$ for this light polarization, optical transitions in which are allowed by the selection rules. As soon as we obtain the interband population inversion, the light becomes amplified. At high carrier densities, the stimulated optical transitions give rise to a high optical gain. It is shown that under the compressive strain, the shift of the valence and conduction bands leads to a blue shift of the gain spectra, with respect of the band-edge of unstrained quantum well.

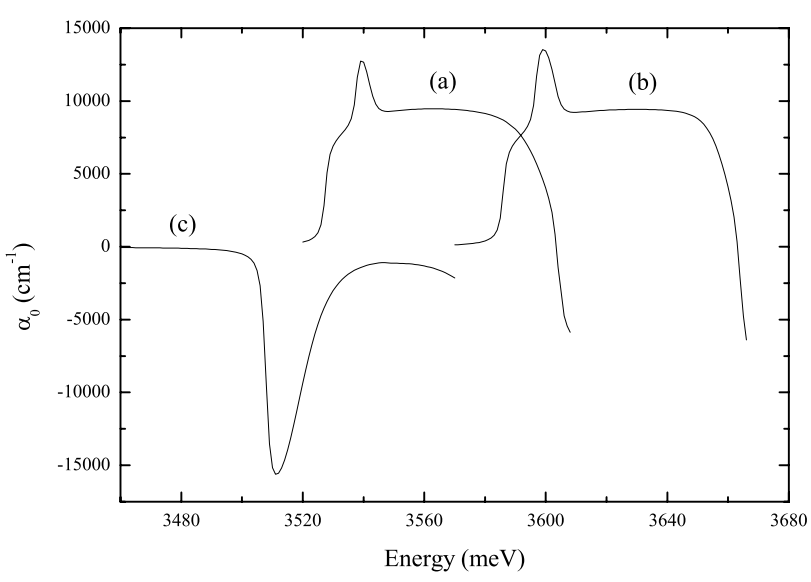

Fig. 4. Light gain coefficient of $\mathrm{GaN} / \mathrm{Al}_{0.19} \mathrm{Ga}_{0.81} \mathrm{~N}$ quantum well for: (a) transverse polarization of unstrained quantum well; (b) $x$ (or $y$ ) polarization with the compressive biaxial strain; (c) $z$ polarization with the tensile biaxial strain.

It is found that the effective mass is drastically changed under the tensile strain. This leads to changes of sign and polarization of the gain coefficient, as shown in Fig. 4. It can be explained by appearance of the circular loop with radius $k_{t} \approx 0.7 \mathrm{~nm}^{-1}$ in valence band structure of zinc-blende $\mathrm{GaN}$ quantum well under the tensile strain. Extremum loop effects in the band structure of würtzite symmetry, in which the linear term in wave vector exists, are known from papers [35]. With appearance of the spectrum region characterized with a negative effective mass at the Brillouin zone center, holes are located on the loop of valence-band maxima. As a consequence of this, the optical transitions near the band-edge occur with high light absorption suppressing the laser effect. Under the tensile strain, the shift of the valence and conduction bands leads to a red shift of the absorption spectra, with respect of the band-edge of unstrained quantum well.

In both zinc-blende and würtzite crystal structures, each atom is surrounded by four nearest neighbors forming an ideal tetrahedron. The valence electrons of this crystal structure form hybridized $s p^{3}$ orbitals [20].
This $s p^{3}$ hybridization is well known from the bonding of a methane molecule. It is interesting to consider the analogy existing between the dependence of the matrix elements on the strain under the lattice mismatch and strain effects on behavior of the bond angles of the tetrahedron. It is known [36] that in the case of tensile biaxial strain the bonding tetrahedra are compressed along the $c$ axis by shrinking the distance between the Ga-N layers toward a planar structure, which changes the bond angles. This causes dehybridization from $s p^{3}$ hybrids towards $s p^{2}$ and $p_{z}$ orbitals. The quantum mechanical problem of dehybridization $s p^{3}$ hybrids towards $s p^{2}$ and $p_{z}$ orbitals is consistent with a tendency of an increase of the $|Z\rangle$ state in the light hole band under the tensile biaxial strain, which yields the matrix elements for the $z$ polarization. It is shown in Fig. 3c.

We have investigated the strain effects on the valence band structures, the interband matrix elements, and the light gain spectrum in the pseudomorphically strained zinc-blende nitride quantum well. With this purpose, we use a $3 \times 3$ block Hamiltonian to calculate the valence band spectra in the quantum well heterostrusture. A detailed analysis is presented for the dependence of the hole spectra, the matrix elements, and light gain spectra on strain under the lattice mismatch in the heterostructures. The analysis of the band structure of quantum well under the compressive strain exhibits a downward shift of the valence bands. For the quantum well with the compressive strain $\varepsilon_{x x}=-0.54 \%$, the matrix elements for transitions from the first hole band have the strict $x$ (or $y$ ) light polarization, while with the tensile strain $\varepsilon_{x x}=0.54 \%$, the considered matrix elements have the strict $z$ light polarization. Under the tensile strain, both the large negative mass and the strong modification of the matrix elements arise at the Brillouin zone center. The casual twofold degeneration of double spin degenerated heavy hole and light hole states arises at the Brillouin zone center. An increase of the $|Z\rangle$ state in the light hole band under the tensile strain is found. It yields the strong matrix element for the $z$ polarization. The circular loop of valence band maxima with the finite radius in the heterostructure under the tensile strain is found. Under the compressive strain, holes are located on the upper valence subbands, while under the tensile strain holes are located on the circular loop of the valence band maxima. It is shown that laser effect is suppressed in GaN quantum well with the tensile strain $\varepsilon_{x x}=0.54 \%$, while at $\varepsilon_{x x}=-0.54 \%$ the stimulated optical transitions give rise to a high optical gain. In the paper, importance of the extremum loop of valence band in zinc-blende GaN quantum well under the tensile strain is found. Although the extremum loop effects in würtzite crystals were studied [35], suppression of the laser effects was not indicated in publications. It is found that the internal strain effects play a significant role in optical properties of the quantum well heterostructures. 
The author acknowledges many helpful discussions with Prof. V.A. Kochelap and Prof. V.I. Sheka.

\section{References}

1. S. Nakamura, G. Fasol, The Blue Laser Diode. Springer, Berlin, 1997; R.L. Aggarwal, P.A. Maki, Z.-L. Liau, and I. Melngailis // J. Appl. Phys. 79, p. 2148 (1996); S. Nakamura, M. Senoh, S. Nagahama, N. Iwasa, T. Yamada, T. Matsushita, H. Kiyoku, and Y. Sugimoto // Jpn J. Appl. Phys. Part 1, 35, p. $L 74$ (1996); S. Nakamura // J. Vac. Sci. Technol. A 13, p. 705 (1995).

2. N. Savage // Nature Photonics 1, p. 83 (2007).

3. M. Asif Khan et al. // J. Appl. Phys. 76, p. 1161 (2000).

4. M. Iwaya, S. Takanami, A. Miyazaki, Y. Wanatabe, S. Kamiyama, H. Amano, and I. Akasaki // Phys. status solidi (a) 188, p. 117 (2001).

5. G. Kipshidze, V. Kuryatkov, B. Borisov, M. Holtz, S. Nikishin, H. Temkin // J. Appl. Phys. 93, p. 1363 (2003).

6. J.P. Zhang et al. // Appl. Phys. Lett. 81, p. 4910 (2002).

7. V. Adivarahan, S. Wu, J.P. Zhang, R.A. Chitnis, M. Shatalov, V. Mandavilli, R. Gaska, and M.A. Khan // Appl. Phys. Lett. 84, p. 4762 (2004).

8. A. Khan, K. Balakrishnan, T. Katona // Nature Photonics 2, p. 77 (2008).

9. S. Nagahama, T. Yanamoto, M. Sano, and T. Mukai // Jpn J. Appl. Phys. Part 2, 40, p. L788 (2001).

10. S. Nagahama, T. Yanamoto, M. Sano, and T. Mukai // Jpn J. Appl. Phys. Part 1, 41, p. 5 (2002).

11. M. Kneissl, D. Treat, M. Teepe, N. Miyashita, and N.M. Johnson // Appl. Phys. Lett. 82, p. 2386 (2003).

12. M. Kneissl, D. Treat, M. Teepe, N. Miyashita, and N.M. Johnson // Appl. Phys. Lett. 82, p. 4441 (2003).

13. S. Masui, Y. Matsuyama, T. Yanamoto, T. Kozaki, S. Nagahama, and T. Mukai // Jpn J. Appl. Phys. Part 2, 42, p. L1318 (2003).

14. K. Iida et al. // Jpn J. Appl. Phys. Part 2, 43, p. L499 (2004).

15. T. Takano, Y. Narita, A. Horiuchi, and H. Kawanishi // Appl. Phys. Lett. 84, p. 3567 (2004).

16. H. Markoc., S. Strite, G.B. Gao, M.E. Lin, B. Sverdlov, and M. Burns // J. Appl. Phys. 76, p. 1363 (1994).

17. W.G. Perry, T. Zheleva, M.D. Bremser, R.F. Davies, W. Shan, and J.J. Song // J. Electron. Mater. 26, p. 224 (1997).
18. S.C. Jain, M. Willander, J. Narayan, and R. Van Overstraeten // J. Appl. Phys. 87, p. 965 (2000).

19. Semiconductors, edited by O. Madelung. Springer, Berlin, 1991; W. Shan, T.J. Schmidt, X.H. Yang, S.J. Hwang, J.J. Song, and B. Goldenberg // Appl. Phys. Lett. 66, p. 985 (1995).

20. P.Y. Yu, M. Cardona, Fundamentals of Semiconductors. Springer, Berlin, 1996.

21. G.L. Bir, G.E. Pikus, Symmetry and Strain-Induced Effects in Semiconductors. Wiley, New York, 1974.

22. E.I. Rashba, Fiz. Tverd. Tela 1, p. 407 (1959) (in Russian) [Sov. Phys. Solid State 1, p. 368 (1959)]; E.I. Rashba and V.I. Sheka // Ibid., Collection of Papers, p. 162 (1959); G.E. Pikus // Zhurnal experim. teoret. fiziki 41, p. 1258 (1961) (in Russian) [Sov. Phys. JETP 14, p. 898 (1962)].

23. Yu.M. Sirenko, J.-B. Jeon, K.W. Kim, M.A. Littlejohn, M.A. Stroscio // Phys. Rev. B 53, p. 1997 (1996).

24. J.M. Luttinger // Phys. Rev. 102, p. 1030 (1956).

25. S. Y.-P. Chao, S.L. Chuang // Phys. Rev. B 46, p. 4110 (1992).

26. S.L. Chuang, C.S. Chang // Phys. Rev. B 54, p. 2491 (1996).

27. W. J. Fan, M.F. Li, T.C. Chong, and J.B. Xia // J. Appl. Phys. 79, p. 188 (1996).

28. C.-H. Kim, B.-H. Han // Solid State Communs 106, p. 127 (1998).

29. M. Suzuki, T. Uenoyama // Appl. Phys. Lett. 69, p. 3378 (1996).

30. Yu.M. Sirenko, J.-B. Jeon, K.W. Kim, M.A. Littlejohn, M.A. Stroscio // Appl. Phys. Lett. 69, p. 2504 (1996).

31. L.D. Landau, E.M. Lifshits, Quantum Mechanics. Pergamon, Oxford, 1977.

32. R.C. Casella // Phys. Rev. 114, p. 1514 (1959).

33. S.L. Chuang // J. Quantum Electron. 32, p. 1791 (1996).

34. T. Yamada, D.K. Ferry // Solid State Electronics 38, p. 881 (1995).

35. R.C. Casella // Phys. Rev. Lett. 5, p. 371 (1960); E.I. Rashba // Fiz. Tverd. Tela 2, p. 1224 (1960) (in Russian) [Sov. Phys. Solid State 2, p. 1109 (1960)]; G.D. Mahan and J.J. Hopfield // Phys. Rev. A 135, p. 428 (1964); L.C. Voon et al. // Phys. Rev. B 53, p. 10703 (1996).

36. J.-M. Wagner, F. Bechstedt // Phys. Rev. B 66, 115202 (2002). 